\title{
Reliability Estimation of Fatigue Damaged Structure using Various Probability Theories*
}

\author{
Ouk Sub LEE**, Seon Soon CHOI*** and Dong Hyeok KIM**** \\ **School of Mechanical Engineering, INHA University \\ 253 Yonghyun-dong, Nam-gu, Incheon, 402-751, Korea \\ *** Department of Car Mechatronics, SAHMYOOK University \\ 26-21 Gongneung-2dong, Nowon-gu, Seoul, 139-742, Korea \\ Corresponding E-mail: choiss@syu.ac.kr \\ **** Department of Mechanical Engineering, INHA University \\ 253 Yonghyun-dong, Nam-gu, Incheon, 402-751, Korea
}

\begin{abstract}
The methodologies to calculate failure probability and to estimate the reliability of fatigue loaded structures are developed. The applicability of the methodologies is evaluated with the help of the fatigue crack growth models suggested by Paris and Walker. The probability theories such as the FORM (first order reliability method), the SORM (second order reliability method) and the MCS (Monte Carlo simulation) are utilized. It is found that the failure probability decreases with the increase of the design fatigue life, and the decrease of the initial edge crack size, the stress range, and the stress ratio. Furthermore, according to the sensitivity analysis of random variables, it is found that the slope of Paris equation affects the failure probability dominantly among other random variables in the Paris and the Walker models.
\end{abstract}

Key words: Reliability, FORM(First Order Reliability Method), SORM(Second Order Reliability Method), MCS(Monte Carlo Simulation), Fatigue, Failure Probability, Sensitivity, Paris Equation, Walker Equation

\section{Introduction}

The repeated loads may lead to failure of material even when the load level is lower than the ultimate limit states. Many mechanical structures such as train axles and wheels, load bearing parts of automobiles, offshore structures, and bridges are designed to endure for a long term up to giga-cycle loadings in the actual service. Furthermore, some mechanical structures in the various areas are needed to be investigated if the operation life can be extended beyond the design life because of the economic consideration. In such circumstances, mechanical components of these structures are exposed to tremendous number of stress/strain cycles in the long term service. Thus, the fatigue property of the structural materials under the long term cyclic loadings is an important subject to provide the safety design data for such mechanical structures ${ }^{(1)-(3)}$.

In the fatigue design, the use of S-N curves is well established. These curves predict fatigue failure under constant amplitude loading, but cannot incorporate information related to crack detection and/or measurement. As a result, the structures must be repaired, if the crack is discovered. However, the use of fracture mechanics techniques can be successfully applied to this problem. The fracture mechanics needs the information about the defects, or cracks to be used in the analysis. Since the size and location of defects are quite random, the deterministic analysis may provide incomplete results about the structure safety. Also the randomness of loads, geometry and material properties influence significantly the reliability 
of a structure. Therefore, the fracture mechanics with a probabilistic method is thought to be an appropriate approach to provide a useful tool to solve these problems ${ }^{(4)-(8)}$.

With regard this point, we make our efforts to propose a reliability estimation methodology for fatigue damaged structure using the probabilistic theory. An iteration method to calculate the reliability index, which is proposed by Rackwitz and Fiessler is utilized to accomplish our goals. Moreover, the failure probability using the SORM has been carried out to make up for the FORM's weakness. This point is considered as the discrimination from other published researches. Furthermore, the validity of the proposed methodology was verified by making a comparison between the results of this paper with ones obtained from the Monte Carlo simulation (MCS).

Two fatigue crack propagation models suggested by Paris and Walker are used to formulate the limit state function for assessing the failure of fatigue loaded structures in this paper. The sensitivity of each random variable, which is quantifying the effect on the failure probability, is also estimated. And the results for the case study are systematically investigated to assess the accuracy of the reliability with the help of the FORM, the SORM and the MCS. Based on the obtained output, it is strongly recommended that the proposed methodology may be applied into the realistic problem with replacing the parameters as real values.

\section{Fatigue Models}

The strength of a component or structure can be significantly reduced by the presence of a crack. The fatigue crack growth rate, $d a / d N$, versus the applied stress intensity factor range, $\Delta K$ can be obtained from fatigue crack propagation experiments. The corresponding applied stress intensity factor range, $\Delta K$, is calculated when the crack length, $a$ and the applied stress range, $\Delta S$, are measured in the experiments as below ${ }^{(1),(3),(4)}$.

$$
\begin{aligned}
\Delta K_{I}=\Delta K & =K_{\max }-K_{\min }=S_{\max } \sqrt{\pi a} \alpha-S_{\min } \sqrt{\pi a} \alpha \\
& =\left(S_{\max }-S_{\min }\right) \sqrt{\pi a} \alpha=\Delta S \sqrt{\pi a} \alpha
\end{aligned}
$$

Where $\alpha$ is the geometry factor. Since the stress intensity factor is undefined in the compression, $K_{\min }$ is taken as zero if $S_{\min }$ is compressive. The correlation for constant amplitude loading is usually a log-log plot of the fatigue crack growth rate, $d a / d N$, in $m /$ cycle, versus the opening mode stress intensity factor range, $\Delta K_{I}$ (or $\Delta K$ ), in $\operatorname{MPa} \sqrt{m}$.

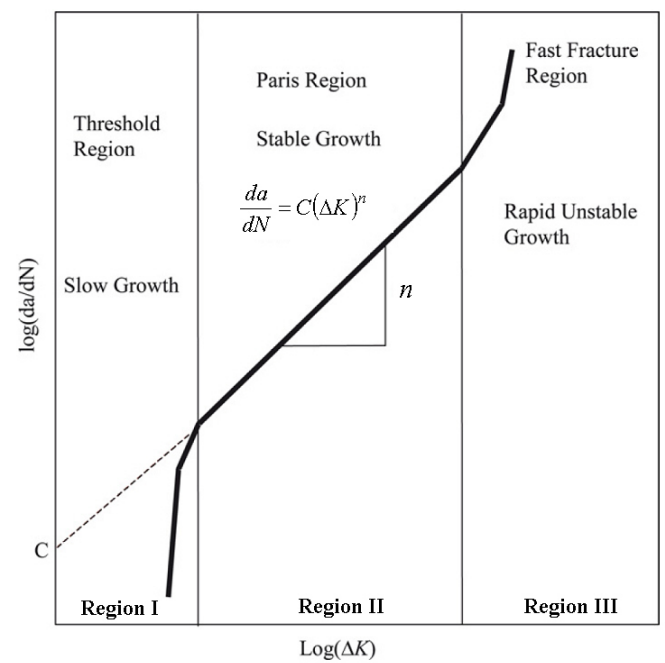

Fig. 1 Schematic behavior of fatigue crack growth rate versus stress intensity factor range. 
The typical log-log plot of fatigue crack growth rate versus stress intensity factor range as shown schematically in Fig. 1 has a sigmoid shape that can be divided into three major regions. Region $\mathrm{I}$ is the near threshold region and indicates a threshold value, $\Delta K_{t h}$, and there is no observable crack growth below this value. This threshold occurs at crack growth rates on the order of $1 \times 10^{-10} \mathrm{~m} / \mathrm{cycle}$ or less. Region II shows essentially a linear relationship between $\log d a / d N$ and $\log \Delta K$, which corresponds to the formula suggested by Paris ${ }^{(1),(3),(4)}$.

$$
\frac{d a}{d N}=C(\Delta K)^{n}
$$

Where $n, C$ are material constants. $n$ is the slope of the line and $C$ is the coefficient found by extending the straight line to $\Delta K=1 M P a \sqrt{m}$. Region II fatigue crack growth corresponds to stable macroscopic crack growth that is typically controlled by the environment. Microstructure and mean stress have less influence on fatigue crack growth behavior in region II than in region I. In region III, the fatigue crack growth rates are very high as it approaches instability, and little fatigue crack growth life is involved. This region is controlled primarily by fracture toughness $K_{C}$ or $K_{I C}$, which depends on the microstructure, mean stress, and environment.

Conventional S-N or $\varepsilon-\mathrm{N}$ fatigue behavior is usually referenced to the fully reversed stress or strain conditions $(R=-1)$. However, fatigue crack growth datas are usually referenced to the pulsating tension condition with $R=0$ or approximately zero.

The general influence of mean stress on fatigue crack growth behavior can be estimated by using the stress ratio, $R=K_{\min } / K_{\max }=S_{\min } / S_{\max }$, which is used as the principal parameter and has the positive value, $R \geq 0$. It should be recognized that the effect of the $R$ ratio on the fatigue crack growth behavior is strongly material dependent.

A common empirical relationship used to describe mean stress effects with $R \geq 0$ is the Walker equation as below ${ }^{(1),(3)-(5)}$.

$$
\frac{d a}{d N}=\frac{C(\Delta K)^{n}}{(1-R)^{n(1-\lambda)}}=C^{\prime \prime}(\Delta K)^{n}
$$

Where $C$ and $n$ are the coefficient and slope of Paris equation for $R=0$, respectively, and $\lambda$ is a material constant. Paris equation and Walker equation are basically similar, with different coefficients of the equations, $C$ and $C^{\prime \prime}$, as below.

$$
C^{\prime \prime}=\frac{C}{(1-R)^{n(1-\lambda)}}
$$

Because the effect of $R$ on fatigue crack growth is known as material dependent, it is necessary to determine the material constant, $\lambda$. Value of $\lambda$ for various metals ranges from 0.3 to nearly 1 , with a typical value of around 0.5 .

\section{Probability Theory}

\subsection{FORM (first order reliability method)}

The failure probability is calculated by using the FORM, which is one of the methods utilizing the reliability index. The FORM method is based on the first-order Taylor series approximation of a limit state function (LSF), which is defined as below ${ }^{(6)-(11)}$.

$$
Z=R E-L O
$$

Where, $R E$ is the resistance normal variable, and $L O$ is the load normal variable. Assuming that $R E$ and $L O$ are statistically independent, normally distributed random variables, the variable $Z$ is also normally distributed. The failure occurs when $R E<L O$, i.e., $Z<0$. The failure probability is given as below. 


$$
\begin{aligned}
& P F=P[Z<0]=\int_{-\infty}^{0} \frac{1}{\sigma_{Z} \sqrt{2 \pi}} \exp \left\{-\frac{1}{2}\left(\frac{Z-\mu_{Z}}{\sigma_{Z}}\right)^{2}\right\} d Z \\
& =\int_{-\infty}^{-\beta} \frac{1}{\sqrt{2 \pi}} \exp \left\{-\frac{U^{2}}{2}\right\} d U=\Phi(-\beta)
\end{aligned}
$$

Where $\mu_{Z}$ and $\sigma_{Z}$ are the mean and standard deviation of the variable $Z$, respectively, and $\Phi$ is the cumulative distribution function for a standard normal variable, and $\beta$ is the safety index or reliability index and the coefficient of variation (C.O.V) denoted as below.

$$
\beta=\frac{\mu_{Z}}{\sigma_{Z}}=\frac{\mu_{R}-\mu_{L}}{\sqrt{\sigma_{R}^{2}+\sigma_{L}^{2}}} \quad, \quad \text { C.O.V }=\frac{\sigma_{X}}{\mu_{X}}
$$

Equation (7) can be used when the system has a linear LSF. Actually, most real systems and cases do not have linear LSF but rather a nonlinear LSF. So, for a system that has a nonlinear LSF, Eq. (7) cannot be used to calculate the reliability index. Rackwitz and Fiessler proposed a method to estimate the reliability index that uses the procedure shown in Fig. 2 for a system having a nonlinear LSF. In this paper, we iterate the loop, as shown in Fig. 2, to determine a reliable reliability index until the reliability index converges to a desired value $(\Delta \beta \leq 0.001)^{(10),(11)}$.

The LSF must be defined to formulate the FORM and evaluate the reliability. In this paper, the LSF can be defined by using the fatigue models as below ${ }^{(6)-(8)}$.

$$
Z=N_{D}-N_{f}
$$

Where, $N_{D}$ is the design fatigue life and $N_{f}$ is the fatigue life estimated from the fatigue crack growth models such as Paris and Walker models using Eq. (2) or (3).

The sensitivity index, which is used to evaluate the effect of random variables on the failure probability, is denoted as below ${ }^{(10),(11)}$.

$$
S I=\frac{\left(\frac{\partial Z}{\partial X}\right)}{\left(\sqrt{\sum\left(\frac{\partial Z}{\partial X}\right)^{2}}\right)}
$$

Where $\partial Z / \partial X$ is the partial derivative of a random variable $X$.

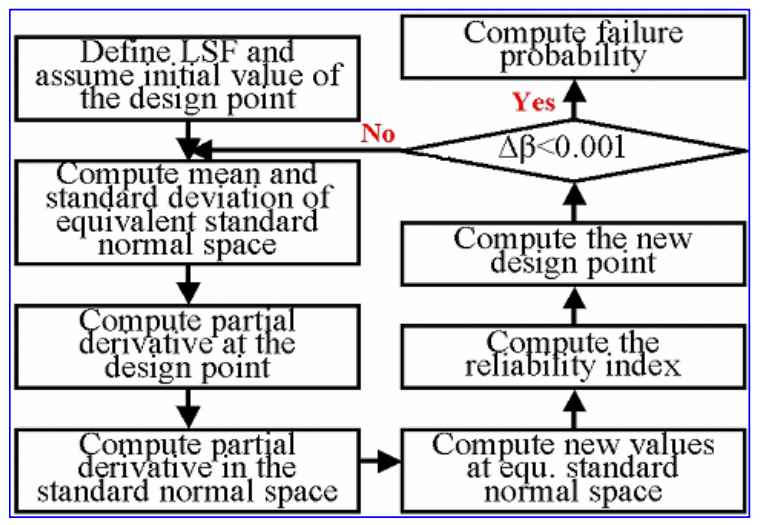

Fig. 2 Computation process of the reliability index. 


\subsection{SORM (second order reliability method)}

The computations required for reliability analysis of systems with linear LSF are relatively simple. However, the LSF could be nonlinear either due to a nonlinear relationship the random variables in the LSF or due to some variables being non-normal.

The FORM approach will give the same reliability index for both linear and nonlinear limit state cases, if the minimum distance point is same. But it is apparent that the failure probability of the nonlinear limit state would be less than that of the linear limit state, due to the difference in the failure domains. The curvature of the limit state around the minimum distance point determines the accuracy of the first order approximation in the FORM. The SORM improves the FORM result by including additional information about the curvature of the limit state.

The SORM approach was first explored by Fiessler using various quadratic approximations. A simple closed form solution for probability computation using a second order approximation and adopting the theory of asymptotic approximation was given by Breitung ${ }^{(6)-(8),(10)}$.

$$
P F_{\text {SORM }}=\Phi(-\beta) \prod_{i=1}^{n-1}\left(1-\beta \kappa_{i}\right)^{-1 / 2}
$$

Where $\kappa_{i}$ denotes the principal curvatures of the LSF at the minimum distance point and $\beta$ is the reliability index calculated by using the FORM. The principal curvatures are computed by using steps shown in Fig. 3 .

\subsection{MCS (Monte Carlo simulation)}

Unlike many engineering analytical results, the ones obtained by probabilistic methods are difficult to verify experimentally. However, the adequacy of the results out of the FORM and the SORM may be required to be verified somehow. We use the MCS technique to do this job performed by the steps shown in Fig. $4^{(6)-(8),(10),(11)}$.

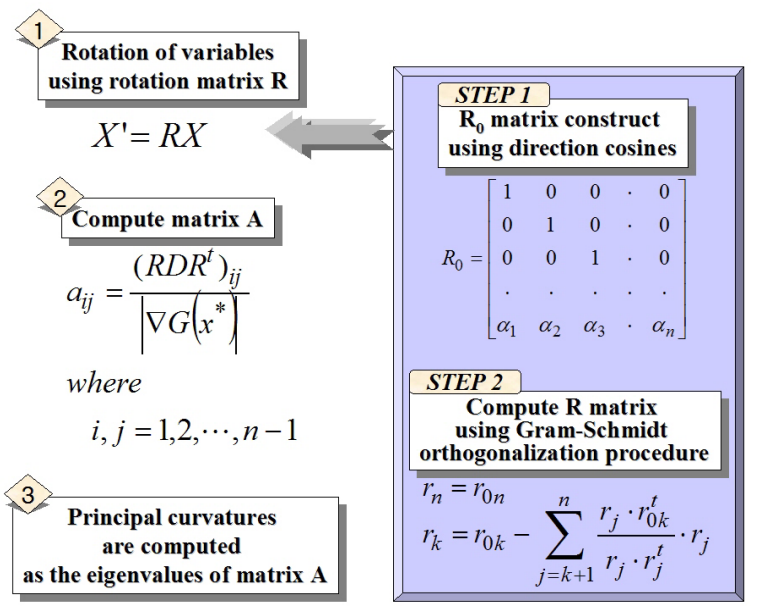

Fig. 3 Process of computing the principal curvatures.

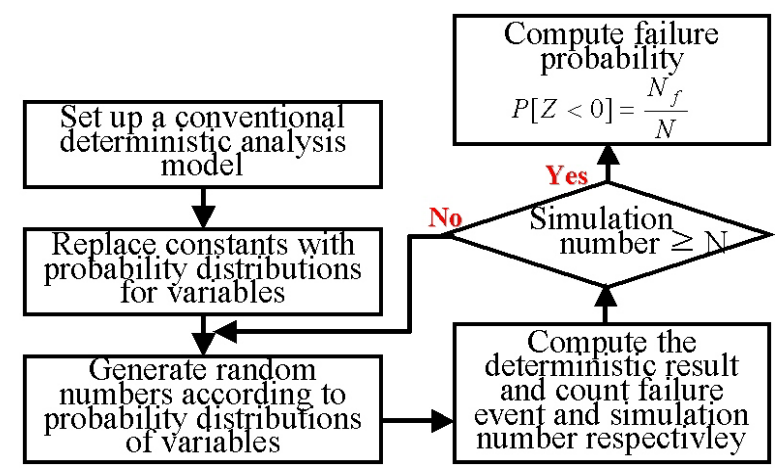

Fig. 4 Computation process of the failure probability by the Monte Carlo simulation. 


\section{A Case Study}

In this paper, we formulate the LSF using the fatigue models, and the failure probability is estimated by using the FORM and the SORM for fatigue experiment data with a single edge crack shown in Fig. 5. The specimen is a very wide SAE 1020 cold-rolled thin plate subjected to constant amplitude uniaxial cyclic loads. The random variables and their values to apply at fatigue models are listed in Table $1^{(1)-(5)}$.

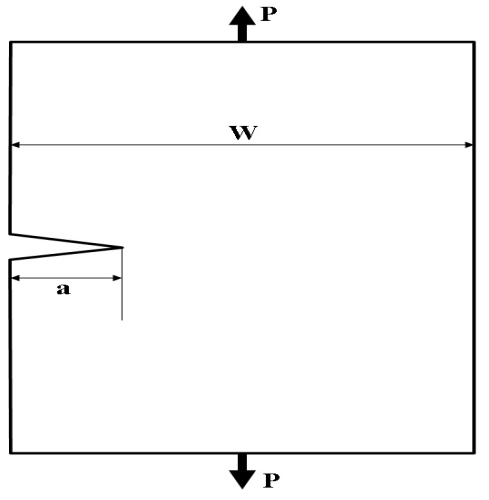

Fig. 5 The geometry of single edge crack specimen.

Table 1. Random variables and its statistical values used in a case study.

\begin{tabular}{c|c|c}
\hline Variable & Mean & C.O.V \\
\hline \multirow{2}{*}{$S_{\max }$} & $200 \mathrm{MPa}$ (Paris) & 0.002 \\
\cline { 2 - 3 }$S_{\min }$ & $300 \mathrm{MPa}$ (Walker) & 0.002 \\
\cline { 2 - 3 } & $-50 \mathrm{MPa}$ (Paris) & 0.002 \\
\hline \multirow{2}{*}{$S_{y}$} & $100 \mathrm{MPa}$ (Walker) & 0.002 \\
\hline$S_{u}$ & $630 \mathrm{MPa}$ & - \\
\hline$E$ & $670 \mathrm{MPa}$ & - \\
\hline \multirow{2}{*}{$K_{c}$} & $207 \mathrm{GPa}$ & - \\
\hline \multirow{2}{*}{$a_{i}$} & $104 \mathrm{MPa} \sqrt{m}$ & 0.01 \\
\hline \multirow{2}{*}{$a_{f}$} & $0.001 \mathrm{~m}$ & 0.01 \\
\cline { 2 - 3 } & $0.068 \mathrm{~m}$ (Paris) & 0.01 \\
\hline$\alpha$ & 1.12 & - \\
\hline$C$ & $6.9 \times 10^{-12}$ & 0.02 \\
\hline \multirow{2}{*}{$n$} & 3.0 & 0.02 \\
\hline \multirow{2}{*}{$N_{D}$} & $129000 \mathrm{cycle}$ (Paris) & 0.003 \\
\cline { 2 - 3 } & $65000 \mathrm{cycle}$ (Walker) & 0.003 \\
\hline$\lambda$ & 0.5 & - \\
\hline
\end{tabular}

\section{Results and Discussion}

In this paper, the LSF is formulated by using the fatigue models suggested by Paris and Walker. And the failure probability is estimated by using the values of random variables and probability theories such as the FORM, the SORM and the MCS. The relationship between failure probability and variation of random variables is shown in Fig. 6 corresponding to the fatigue models and the probability theories. It is found from Fig. 6 that the failure probability decreases with the increase of the design fatigue life, and the decrease of initial edge crack size, the stress range, and the stress ratio. The specific statistical values are used in a deterministic case study to compare the results out of the Paris model to the Walker model. 


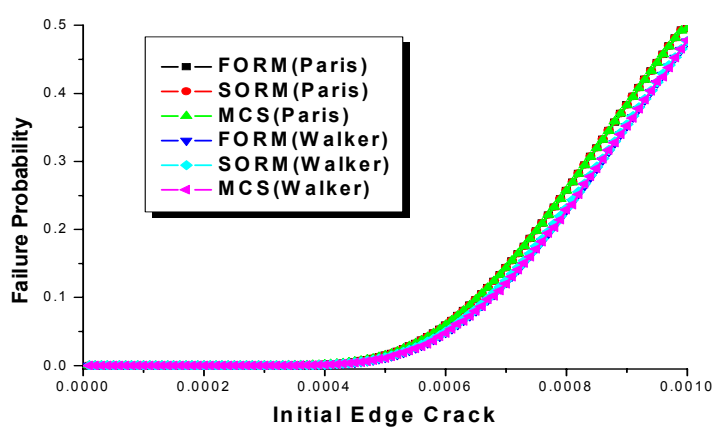

(a) initial edge crack

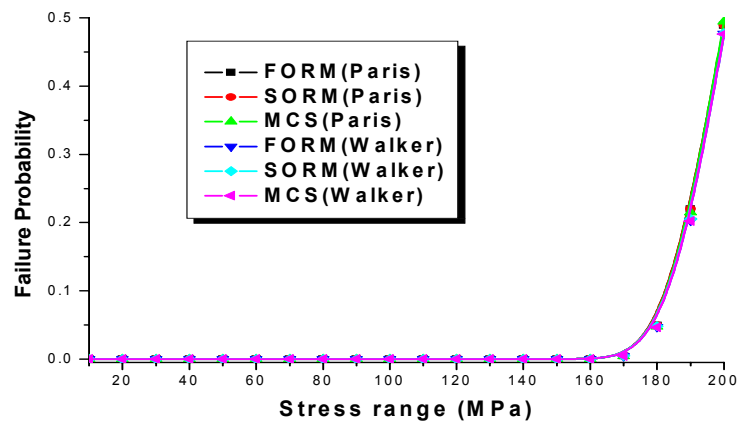

(b) stress range

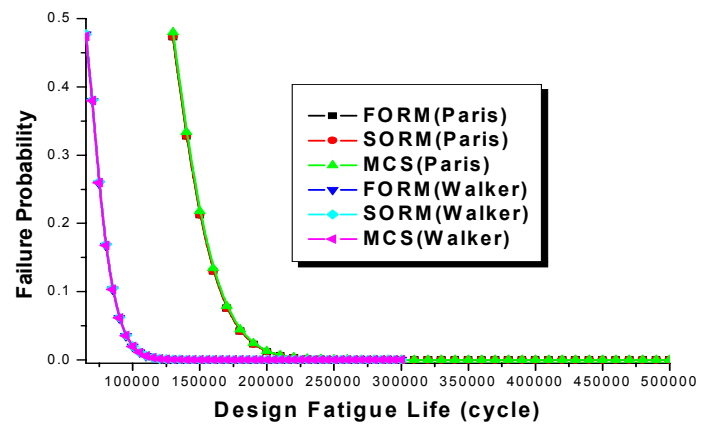

(c) design fatigue life

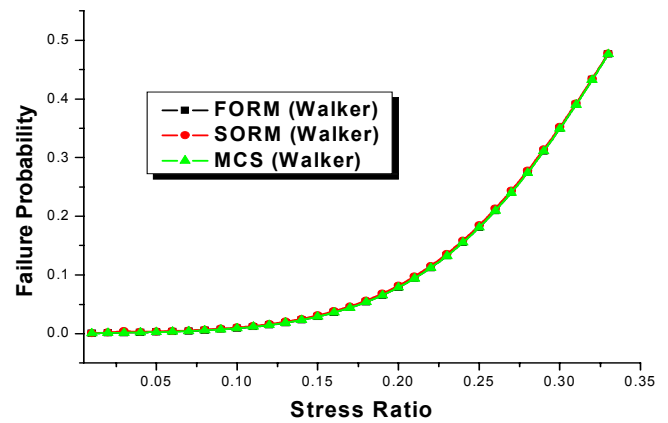

(d) stress ratio

Fig. 6 Relationship between failure probability and various random variables according to the FORM, the SORM and the MCS. 
It is found in Fig. 6 that the failure probabilities based on the Paris and Walker models are turned out to be very similar for variation of the initial edge crack size and the stress range. However, the Paris and Walker models show the different failure probabilities with variation of the design fatigue life, because they have different fatigue lives corresponding to the maximum and minimum stresses.

The failure probability of Paris model with the variation of stress ratio isn't shown in Fig. 6(d), since Paris model doesn't consider the stress ratio.

Table 2 quantitatively shows the mean percentile differences among the results of the FORM, the SORM and the MCS for Paris and Walker models, respectively. It is recognized for the Paris model from Table 2 that the FORM and the SORM show the similar failure probability for varying random variables. And it is found that the differences of failure probability between the FORM and the MCS are similar than differences between the SORM and the MCS.

Table 2. Comparison of the mean percentile differences among results obtained by using the FORM, the SORM and the MCS.

\begin{tabular}{|c|c|c|c|c|}
\hline & & $\begin{array}{c}\text { FORM } \\
\text { Vs. } \\
\text { MCS } \\
{[\%]}\end{array}$ & $\begin{array}{c}\text { SORM } \\
\text { vs. } \\
\text { MCS } \\
{[\%]}\end{array}$ & $\begin{array}{c}\text { FORM } \\
\text { vs. } \\
\text { SORM } \\
{[\%]}\end{array}$ \\
\hline \multirow{3}{*}{$\begin{array}{l}\text { Paris } \\
\text { Model }\end{array}$} & $\begin{array}{l}\text { Initial Edge } \\
\text { Crack }\end{array}$ & 2.8523 & 2.8523 & 0.00166 \\
\hline & $\begin{array}{l}\text { Stress } \\
\text { Range }\end{array}$ & 3.2728 & 3.2729 & 0.00046 \\
\hline & $\begin{array}{c}\text { Design } \\
\text { Fatigue Life }\end{array}$ & 10.5702 & 10.5701 & 0.00038 \\
\hline \multirow{4}{*}{$\begin{array}{l}\text { Walker } \\
\text { Model }\end{array}$} & $\begin{array}{c}\text { Initial Edge } \\
\text { Crack }\end{array}$ & 3.9644 & 6.1284 & 23.554 \\
\hline & $\begin{array}{l}\text { Stress } \\
\text { Range }\end{array}$ & 0.8905 & 1.0477 & 3.7256 \\
\hline & $\begin{array}{c}\text { Design } \\
\text { Fatigue Life }\end{array}$ & 6.2603 & 6.6719 & 2.5442 \\
\hline & Stress Ratio & 0.3683 & 17.3519 & 16.9643 \\
\hline
\end{tabular}

It is also recognized from Table 2 for the Walker model that the differences of failure probability between the FORM and the MCS are much similar as those of between the FORM and the SORM, and between the SORM and the MCS.

Although the differences among results for the variation of initial edge crack and the stress ratio are large, the differences are not distinguished clearly in Fig. 6, because the absolute values of the failure probability estimated by the FORM, the SORM and the MCS are very small.

The effects of each random variable on the failure probability are shown in Fig. 7 as the sensitivity index. It is recognized that the slope of Paris equation, $n$, affects dominantly on the failure probability with the variation of the initial edge crack size, the design fatigue life and the stress ratio in the Paris and Walker models. However, it is found that the effects of the slope of Paris equation, $n$, on the failure probability become larger with increases of the stress range, on the other hand, the effects of other random variables such as the coefficient of Paris equation, $C$, the stress range, $\Delta S$, the initial edge crack size, $a_{i}$, the final edge crack size, $a_{f}$, and the design fatigue life, $N_{f}$, on the failure probability become smaller with increases of the stress range. Therefore, it is essential that the material constant such as the slope of Paris equation, $n$, and the coefficient of Paris equation, $C$, must be estimated very carefully using the appropriate method from fatigue experiment.

And it is found from Fig. 7 that the effects of random variable on the failure probability don't vary before and after iteration with the change of design fatigue life and the stress 
ratio in the Paris and Walker models. However, the effects of some random variables on the failure probability become larger or smaller slightly before and after iteration with the variation of the initial edge crack size and the stress range in the Paris and Walker models.

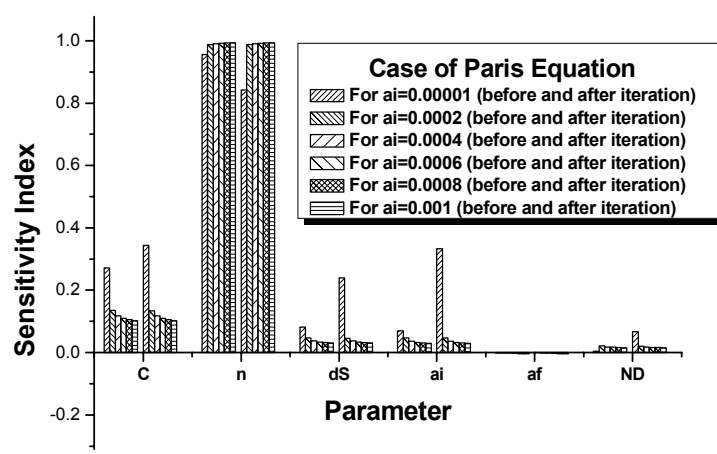

(a) change of initial edge crack

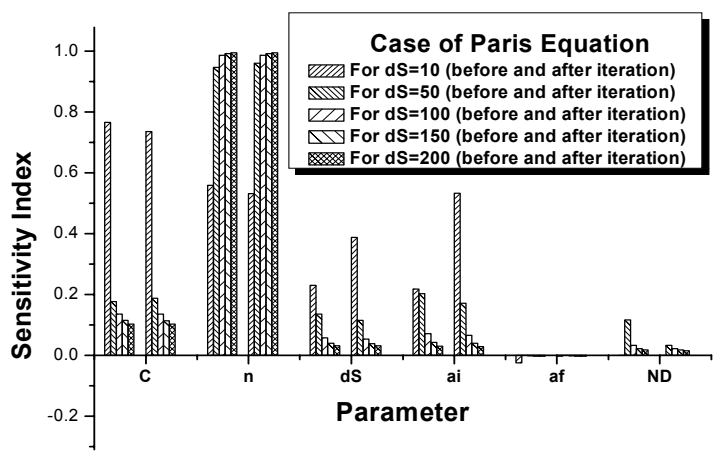

(b) change of stress range

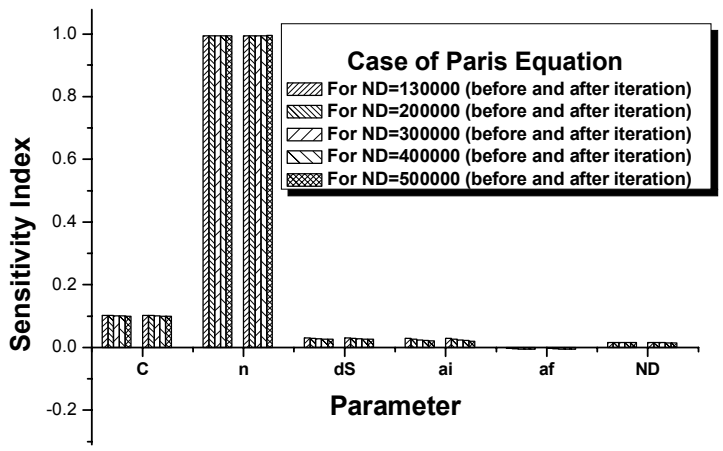

(c) change of design fatigue life 


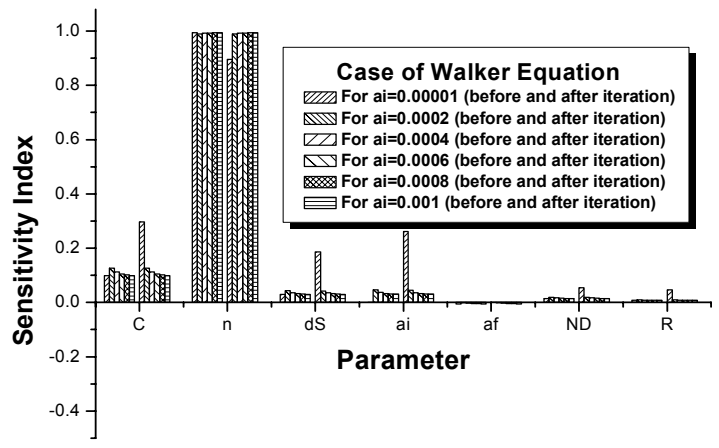

(d) change of initial edge crack

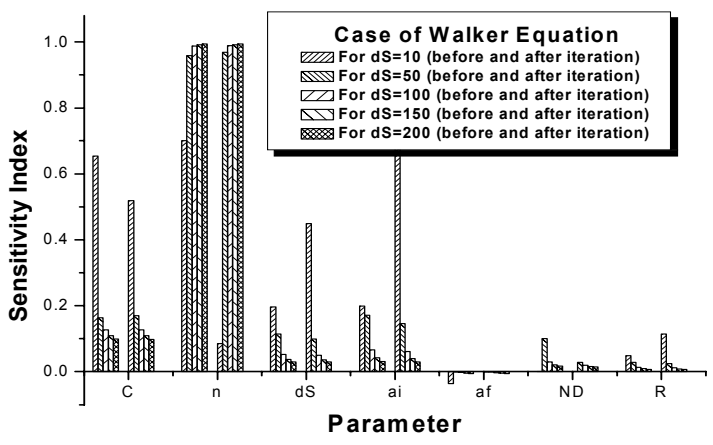

(e) change of stress range

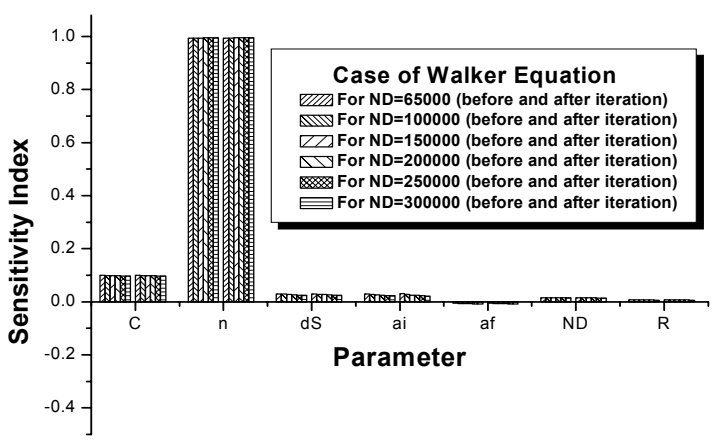

(f) change of design fatigue life

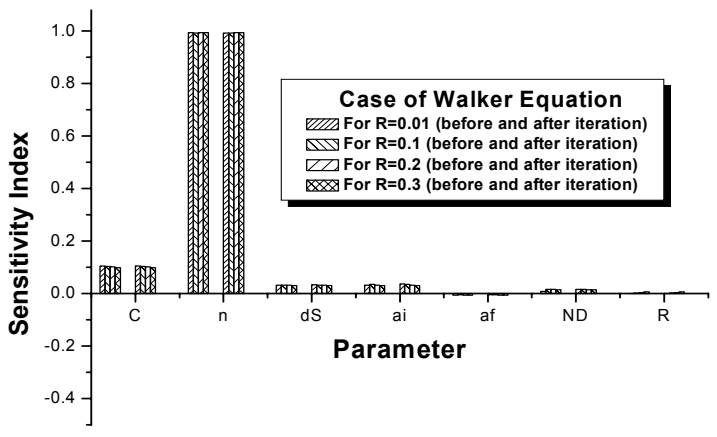

(g) change of stress ratio

Fig. 7 Sensitivity of parameters according to the variation of random variables about Paris and Walker models 


\section{Conclusions}

In this paper, the fatigue crack growth models suggested by Paris and Walker are used to formulate the limit state function (LSF) and the FORM (first order reliability method) and the SORM (second order reliability method) are used to estimate the failure probability. And the MCS (Monte Carlo simulation) and case study are used to evaluate the applicability of the FORM and the SORM by comparing the failure probability. Moreover, the effects of various random variables on the failure probability are systematically studied using the sensitivity index.

The results obtained in this paper are summarized as follows:

1. It is found that the failure probability decreases with the increase of the design fatigue life, and the decrease of the initial edge crack size, the stress range and the stress ratio.

2. It is recognized that the FORM and the SORM show the similar failure probability in the Paris model, and the FORM and the MCS show the similar failure probability in the Walker model, respectively.

3. It is recognized that the slope of Paris equation, $n$, affects dominantly on the failure probability with the variation of random variables in the Paris and Walker models.

4. However, it seems that further studies for more realistic problems are needed to be done in near future and the outcomes should be included in the next publication.

\section{Acknowledgments}

This work was supported by Brain Korea 21 project in 2007 and by Sahmyook Univ. Research Fund.

\section{References}

(1) Stephens, I. S., Ali, F., Robert, R. S. and Henry, O. F., Metal Fatigue in Engineering, John Wiley \& Sons (2001).

(2) Cheung, M. S. and Li, W. C., Probabilistic fatigue and fracture analyses of steel bridges, Structural Safety, 23 (2003), pp. 245-262.

(3) Steven, R. L., Grace, M. D., Faith, R., Randall, L. B., Amy, H., Scott, D. H. and William, W. S., ASM Handbook, Fatigue and Fracture, ASM International Vol. 19(1996).

(4) Anderson, T. L., Fracture Mechanics : Fundamentals and Applications, CRC Press(2005).

(5) Righiniotis, T. D. and Chryssanthopoulos, M. K., Probabilistic fatigue analysis under constant amplitude loading, Journal of Constructional Steel Research, 59 (2003), pp. 867-886.

(6) Lee, O. S., Kim, D. H. and Choi, S. S., Reliability of Buried Pipeline Using A Theory of Probability of Failure, SOLID STATE PHENOMENA, Vol. 110 (2006), pp. 221-230.

(7) Lee, O. S. and Kim, D. H., The Reliability Estimation of Pipeline Using FORM, SORM and Monte Carlo Simulation with FAD, Journal of Mechanical Science and Technology, Vol. 20, No. 12 (2006), pp. 2124-2135.

(8) Lee, O. S., Choi, S. S. and Kim, D. H., Reliability Estimation of Fatigue Damaged Structure Using Various Probability Theories, Proceedings of The International Conference on Advanced Technology in Experimental Mechanics 2007 (ATEM'07) (2007-9), CD-ROM.

(9) Ahammed, M., Probabilistic estimation of remaining life of a pipeline in the presence of active corrosion defects, International Journal of Pressure Vessels and piping, Vol. 75, No. 4 (1998), pp. 321-329.

(10) Mahadevan, S. and Haldar, A., Reliability Assessment Using Stochastic Finite Element Analysis, John Wiley \& Sons (2000).

(11) Mahadevan, S. and Haldar, A., Probability, Reliability and Statistical Method in Engineering Design, John Wiley \& Sons (2000). 